\title{
G2C2 - I. Homogeneous photometry for Galactic globular clusters in SDSS passbands
}

\author{
Joachim Vanderbeke, ${ }^{1,2 \star}$ Michael J. West,,${ }^{2,3}$ Roberto De Propris, ${ }^{4}$ Eric W. Peng, ${ }^{5,6}$ \\ John P. Blakeslee, ${ }^{7,8}$ Andrés Jordán, ${ }^{9}$ Patrick Côté, ${ }^{7}$ Michael Gregg, ${ }^{10,11}$ \\ Laura Ferrarese, ${ }^{7}$ Marianne Takamiya ${ }^{12}$ and Maarten Baes ${ }^{1}$ \\ ${ }^{1}$ Sterrenkundig Observatorium, Universiteit Gent, Krijgslaan 281 S9, B-9000 Gent, Belgium \\ ${ }^{2}$ European Southern Observatory, Alonso de Córdova 3107, 7630355 Vitacura, Santiago, Chile \\ ${ }^{3}$ Maria Mitchell Observatory, 4 Vestal Street, Nantucket, MA 02554, USA \\ ${ }^{4}$ Finnish Centre for Astronomy with ESO (FINCA), University of Turku, Väisäläntie 20, FI-21500 Piikkiö, Finland \\ ${ }^{5}$ Department of Astronomy, Peking University, Beijing 100871, China \\ ${ }^{6}$ Kavli Institute for Astronomy and Astrophysics, Beijing 100871, China \\ ${ }^{7}$ Herzberg Institute of Astrophysics, National Research Council, Victoria, BC V9E2E7, Canada \\ ${ }^{8}$ Department of Physics and Astronomy, Washington State University, 1245 Webster Hall, Pullman, WA 99163-2814, USA \\ ${ }^{9}$ Instituto de Astrofísica, Facultad de Física, Pontificia Universidad Católica de Chile, Av. Vicuña Mackenna 4860, 7820436 Macul, Santiago, Chile \\ ${ }^{10}$ Department of Physics, University of California, Davis, CA 956160, USA \\ ${ }^{11}$ Institute for Geophysics and Planetary Physics, Lawrence Livermore National Laboratory, L-413, Livermore, CA 94550, USA \\ ${ }^{12}$ Physics and Astronomy Department, University of Hawaii Hilo, Hilo, HI 96720, USA
}

Accepted 2013 October 17. Received 2013 October 17; in original form 2013 May 13

\begin{abstract}
We present $g^{\prime}$ and $z^{\prime}$ aperture photometry for 96 Galactic globular clusters, making this the largest homogeneous catalogue of photometry for these objects in the Sloan Digital Sky Survey (SDSS) filter system. For a subset of 56 clusters, we also provide photometry in $r^{\prime}$ and $i^{\prime}$. We carry out comparisons with previous photometry as well as with the SDSS data set. The data will be useful for a series of applications in Galactic and extragalactic astrophysics. Future papers will analyse the colour-metallicity relation, colour-magnitude diagrams and structural parameters. The compilation of results based on this data set will be collected in the Galactic Globular Cluster Catalog (G2C2).
\end{abstract}

Key words: globular clusters: general.

\section{INTRODUCTION}

Globular clusters (hereafter GCs) formed during the earliest episodes of star formation in galaxies. They are found in all but the smallest dwarf galaxies, with massive galaxies hosting systems of hundreds or thousands of clusters. The properties of GCs appear to be very homogeneous from one galaxy to the other (in terms of colour, luminosity distribution, etc.) and this implies that the formation of these objects has been intimately related to the assembly of their parent galaxies (e.g. Harris 1991). GCs are living fossils of the Universe at high redshift (their mass is similar to the Jeans mass at the epoch of recombination) and therefore give a snapshot of conditions as prevailed at early epochs (see West et al. 2004; Brodie \& Strader 2006 for reviews). The integrated properties of GCs therefore provide us with information on the earliest stages of galaxy formation; the high intrinsic luminosities of clusters mean that they can be studied in detail well beyond the Local Group, while the bright end of the GC luminosity function has been detected around a $z \sim 0.2$ elliptical galaxy (Alamo-Martínez et al. 2013).

Most work in both Galactic and extragalactic GCs is still based on the older photometric systems (such as Johnson-Cousins, Washington, etc.). Several authors have remarked on the lack of calibrating studies of GCs in the Sloan Digital Sky Survey (SDSS) system (Jordán et al. 2005; Sinnott et al. 2010; Peacock et al. 2011; Vickers, Grebel \& Huxor 2012); as most such objects are in the south, the vast majority of Galactic GCs have not been imaged by the SDSS. The latest edition of the Harris (1996) compilation (2010 edition, this is the version we refer to in the remainder of the paper) lists $U B V R I$ colours for about half of the 150 Galactic GCs. Nevertheless, this photometry is inhomogeneous, as it is taken from different papers, using different methods and instruments (including photomultipliers, photographic plates and modern CCDs).

The SDSS (York et al. 2000) has now imaged over a quarter of the northern sky (about 14500 square degrees) in five passbands. Together with upcoming imaging surveys in the south, SDSS will completely replace the older Schmidt plate atlases of the sky, and at the same time provide a standardized system of photometry in 
the optical for astrophysics (theoretically, with calibrators in every field). With this motivation, our team embarked on the Galactic Globular Cluster Catalog (G2C2) project, with an ultimate goal of collecting reliable photometry using the SDSS filter system for a large sample of Galactic GCs. In this first paper, we present $g^{\prime}$ and $z^{\prime}$ magnitudes for about two-thirds of the Galactic GCs and $r^{\prime}$ and $i^{\prime}$ magnitudes for about one-third of all Galactic GCs. Future work will discuss the colour-metallicity relation (see the companion Paper II - Vanderbeke et al. 2014), the colour-magnitude diagrams (CMDs) of these clusters, their spectral energy distributions over two decades in wavelength and the structural parameters of GCs using King models.

Here we discuss the buildup of the photometric data base: imaging of 96 Galactic clusters in at least two SDSS bands (as well as two more for a subset of 56 objects). We describe our observations and basic data reduction: we give details about the samples, determination of cluster centres, aperture photometry, estimation of the sky level, removal of outliers, photometric errors and correction for extinction. To assess the quality of our data, we compare these with previous work and carry out a similar analysis on GCs in common with the SDSS footprint. This paper is organized as follows. Section 2 presents the observations and the basic data reduction. We present the integrated photometry and colours for the Galactic GCs in Section 3. We summarize the results in Section 4.

\section{OBSERVATIONS AND DATA REDUCTION}

\subsection{CTIO}

We selected Galactic GCs from the latest versions of the Harris (1996) catalogue, which includes about 150 GCs. Observations were carried out between 2003 May 10 and 2012 June 9 using the Cerro Tololo Inter-American Observatory (CTIO) 0.9 and $1 \mathrm{~m}$ telescopes with the U.S. Naval Observatory (USNO) $g^{\prime} r^{\prime} i^{\prime} z^{\prime}$ filter set. Because the Galactic bulge and hence the bulk of the Galactic GCs are best observable during the Chilean winter, cirrus and bad weather were a real issue during the observing runs: many nights were totally lost due to clouds or strong winds, while other nights were disturbed by cirrus and were not photometric.

For the results in this paper, we reduced 13 nights of observations. Several clusters were observed multiple times and it became clear that only four nights (all of which used the $0.9 \mathrm{~m}$ telescope, with an instrumental set-up as shown in Table 1) could be considered (largely) photometric. During these nights, we collected $g^{\prime}$ and $z^{\prime}$ observations for $81 \mathrm{GCs}$, about half of which we also observed using $r^{\prime}$ and $i^{\prime}$ filters.

For the vast majority of the clusters, we have $60 \mathrm{~s}$ exposures in $g^{\prime}$ and $z^{\prime}$ taken in 2004 June. During the run performed on 2003 May 10, short (between 5 and $30 \mathrm{~s}$ ) and long (270 s in $g^{\prime}, 410 \mathrm{~s}$ in $z^{\prime}$ ) exposures were obtained. Both shorter and longer exposures were used separately to determine magnitudes. Some of the clus-

Table 1. The instrumental set-up.

\begin{tabular}{ll}
\hline Telescope & CTIO 0.9 m \\
Dates & 2003 May-2012 March \\
Filter set & $g^{\prime} r^{\prime} i^{\prime} z^{\prime}$ \\
Spatial scale & $0.396 \mathrm{arcsec} \mathrm{pixel}{ }^{-1}$ \\
Field size & $13.6 \mathrm{arcmin} \times 13.6 \mathrm{arcmin}$ \\
Gain & $3.0 \mathrm{e}^{-} \mathrm{ADU}^{-1}$ \\
Read-out noise & $5 \mathrm{e}^{-}$ \\
Detector & $2048 \times 2046 \mathrm{Tek} 2 \mathrm{~K} \mathrm{CCD}$ \\
\hline
\end{tabular}

ters have very bright stars close to their centres. These very bright stars saturated the $\mathrm{CCD}$ even for the short exposures. For these clusters (NGC 6397, 47 Tuc, NGC 6121), we obtained additional $1 \mathrm{~s}$ exposures. The observations discussed in this paper were performed between 2003 May 10 and 2005 September 26 under seeing conditions varying between 1 and $\sim 2$ arcsec.

The basic data reduction was performed via a dedicated IDLpipeline developed by our team. The procedure largely follows conventional CCD reduction processes. The bias level was estimated separately for each quadrant of the $\mathrm{CCD}$, by computing the median of the corresponding bias section, which was then subtracted for each quadrant. The frames were then flat-fielded by the median of the twilight flats taken each night and corrected for bad columns. To identify and robustly remove cosmic rays, we used the L.A. Cosmic (imaging version) method (van Dokkum 2001).

One additional complication was the incorrect information in the fit headers of the clusters observed from 2005 onwards. Subasavage (private communication) confirmed that, since the telescope control system upgrade in early 2005, the header values (including RA, Dec., airmass and epoch) are not correct. Based on the coordinates obtained from Harris (1996) and the header values (date and time of observation), we computed automatically the airmasses for the observations taken after 2005. Comparison with observation log sheets showed excellent agreement.

During the course of each observing night, a minimum of several dozen standard stars, selected from Smith et al. (2002), were observed at different airmasses. Photometric calibration (i.e. determination of zero-points, colour terms and atmospheric extinction values, as well as removal of other instrumental signatures) was carried out as in Patat \& Carraro (2001). Foreground (Galactic) extinction was estimated for each position using the most recent values from the recalibration of Schlafly \& Finkbeiner (2011).

\subsection{SDSS Data Release 9}

We also considered a sample of 21 Northern hemisphere clusters from the ninth data release of SDSS (Ahn et al. 2012), 6 of which have also been observed by us with the CTIO $0.9 \mathrm{~m}$ telescope. However, NGC 6838 and NGC 6254 were only partly covered by SDSS and were not included in this study, as our procedure (see below) requires us to cover at least the half-light radius in each object. Bright foreground stars outshine GLIMPSE01, Ko 1 and Ko 2. We do not consider these clusters further.

For some other clusters, several SDSS stripes needed to be assembled into mosaics using Montage, ${ }^{1}$ although this may lead to issues with variable sky levels. Although SDSS data have the considerable advantage of being photometrically homogeneous and uniform, the $53.9 \mathrm{~s}$ standard exposure in SDSS saturates bright red giant branch (RGB) stars in some GCs, an effect which becomes clear when comparing the CMDs and which is further discussed in Section 3.6. Note that the 'SDSS' filters at the Apache Point Observatory $2.5 \mathrm{~m}$ telescope (and the CTIO $0.9 \mathrm{~m}$ ) have significantly different effective central wavelengths from the calibrating filters at the USNO $1 \mathrm{~m}$ telescope, where the $u^{\prime} g^{\prime} r^{\prime} i^{\prime} z^{\prime}$ photometric system was defined (Fukugita et al. 1996) and extended with secondary standards by Smith et al. (2000, 2002). The conversion between the $u^{\prime} g^{\prime} r^{\prime} i^{\prime} z^{\prime}$ and ugriz system is given on the SDSS website. ${ }^{2}$ These result in

\footnotetext{
${ }^{1}$ http://montage.ipac.caltech.edu/

${ }^{2} \mathrm{http}: / /$ www.sdss.org/dr6/algorithms/jeg_photometric_eq_dr1.html
} 


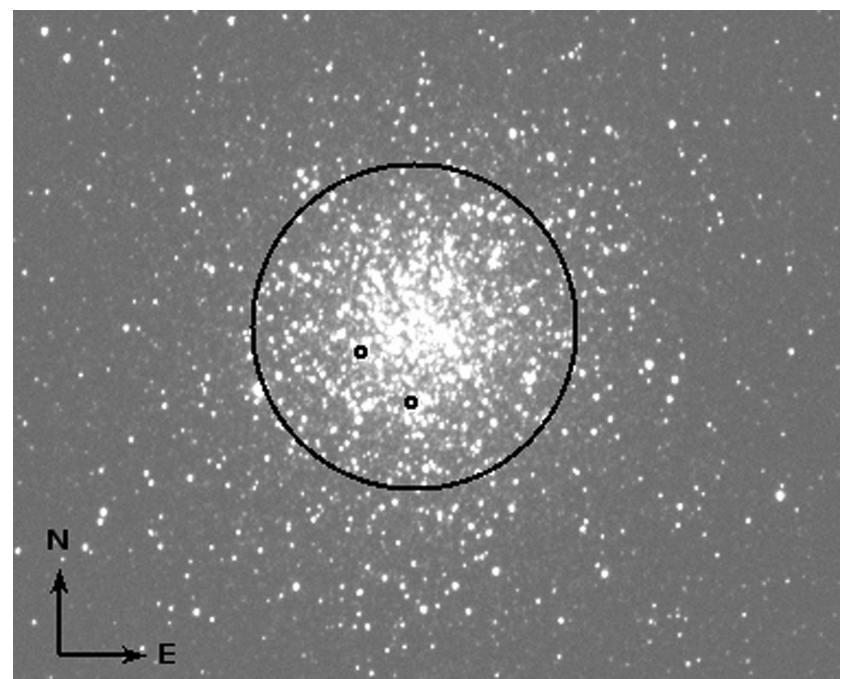

Figure 1. $g^{\prime}$-band observation of NGC 5986. The half-light radius is indicated with the big circle, while the small circles indicate two stars that are likely non-members (contaminants) as selected from their position in the cluster CMD.

negligible changes to the GC colours when compared to the magnitude uncertainties.

For NGC 6341 and NGC 5904, the saturation of the SDSS chip was so severe that almost the entire RGB is brighter than the saturation limit of the CCD. It was nearly impossible to select nonsaturated stars to construct the point spread function (PSF) for the CMDs, which are indispensable in the reduction process (see Section 3.3). We decided to discard these clusters from the sample. Nevertheless, good quality data for NGC 6341 are highly desirable, as this GC is one of the most metal-poor GCs of the Milky Way.

\section{INTEGRATED PHOTOMETRY OF GCS}

Galactic GCs are generally too large on the sky to be completely included within a single CCD frame (see Fig. 1 for an example from our own data). Although we experimented with fitting King models to the surface brightness profiles of the GCs to measure total magnitudes (we discuss this in a subsequent paper), we ultimately chose to derive aperture magnitudes within the clusters' half-light radii (e.g. Peng et al. 2006) to determine integrated colours. As long as the clusters show no strong colour gradients in their outskirts, the integrated colours we present here should be suitable proxies for studies of extragalactic systems as well.

We measured the aperture magnitude within the half-light radius $r_{\mathrm{h}}$ obtained from Harris (1996) where the original values are drawn largely from Trager, Djorgovski \& King (1993), Trager, King \& Djorgovski (1995) and McLaughlin \& van der Marel (2005). As the largest $r_{\mathrm{h}}$ is 5 arcmin, it does not completely fill the CCD field of view and therefore enables us to determine magnitudes for all clusters in a homogeneous manner (as in Peng et al. 2006). To obtain a total magnitude for the clusters, we would need imaging reaching well beyond the tidal radius. However, the largest tidal radius for the Galactic GCs is 53.8 arcmin, much bigger than our field of view. As long as colour gradients in the cluster outskirts are not very strong, the integrated colours determined within the halflight radius aperture should be representative of the total colours. To illustrate this for a cluster with a tidal radius $r_{\mathrm{t}}$ which fits the CTIO $0.9 \mathrm{~m}$ telescope field of view, we compared the $g^{\prime}-z^{\prime}$ colour based on $r_{\mathrm{h}}$ and $r_{\mathrm{t}}$ apertures for NGC 5694. After correcting for contaminants (as will be described in Section 3.3), the colour difference between $r_{\mathrm{h}}$ and $r_{\mathrm{t}}$ apertures is $0.002 \mathrm{mag}$ for this cluster, which is negligible compared to the magnitude uncertainties.

NGC 6287 and NGC 6553 are other clusters with a tidal radius small enough to be entirely covered by the CTIO field of view. However, NGC 6287 was not centred properly on the chip and was not entirely covered as a consequence. The observations of NGC 6553 included some saturated stars within the tidal radius, which is complicating the cleaning of the contaminants and impeding a proper comparison of the colours within the half-light and tidal radii.

Our first step will be to determine the cluster centres for the apertures, followed by estimation of the sky values, removal of contaminants (foreground stars) and measurement of the total flux within the half-light radius. We then discuss extinction, photometric errors and compare our results with previous work and SDSS.

\subsection{Cluster centroids}

Our first step is to determine an accurate cluster centroid for the apertures. For the CTIO data, we followed the method of Bellazzini (2007). We calculated the aperture fluxes in a grid of 25 points around the initial (visual) guess for the cluster centre: the size of the grid is 125 pixels or 60 arcsec. The 'centre' position at which the aperture flux is maximal is adopted as the cluster centroid and used as the reference point for aperture photometry. This was performed separately for all frames, because of inconsistent coordinates in the headers. For the SDSS data, the coordinates listed in Harris (1996) were adopted together with the SDSS astrometric solution. The error in the photometry introduced by the uncertainty in the centre position is estimated in the following way (both for SDSS and CTIO data): magnitudes were computed for apertures centred at four grid points separated by 5 per cent of the stated half-light radius; we calculated the magnitude difference between the aperture magnitudes centred on these positions and the 'true' centre we determined above. The median of this difference is used as the estimate of the uncertainty introduced by the centre determination and is summed in quadrature to the photometric and other errors to obtain the total magnitude error, assuming that these errors are independent. The median contribution of the centre determination to the total magnitude uncertainty is $0.006 \mathrm{mag}$.

To provide a consistency check, we have compared the centroids in the $g^{\prime}$ band to the more accurate central coordinates for each cluster as determined by Goldsbury et al. (2010) with Hubble Space Telescope data. The median difference between both centre determinations is $0.086 r_{\mathrm{h}}$. Using 8.6 per cent $r_{\mathrm{h}}$ instead of 5 per cent $r_{\mathrm{h}}$ to determine the magnitude uncertainty due to the centre determination results in a median additional error of 0.004 mag, which is negligible when compared to the systematic error introduced in Section 3.5.

\subsection{Sky values}

Determination of the sky value proved challenging, as several clusters fill the $0.9 \mathrm{~m} \mathrm{CCD}$ and in most cases the images do not cover the clusters out to their tidal radius. We used мmм (Mean, Median, Mode), a routine available at the IDL astronomy library which was developed to estimate the sky background in a crowded field and was adapted from the DAOPHOT routine with the same name. The algorithm consists of several steps: it first computes the mean and standard deviation of the sky flux, which is used to eliminate outliers. MMM repeats the first step in up to 30 iterations recomputing the sky (eliminating outliers of the previous iteration). As a next 
step, MMM estimates the amount of stellar contamination by comparing the mean, mode and median of the remaining sky pixels. If the mean is less than the mode and the median, then the contamination is slight and the sky is estimated by the mean. If the mean is larger, indicating severe contamination (as the program assumes positive departures from the true sky value in crowded fields), then the true sky value is estimated by $3 \times$ median $-2 \times$ mean. We applied the MMM method to the four corners of each frame in a 100 by 100 pixel area, summing up about 40000 pixels (as some pixels will be identified as outliers by ммм and will not contribute to the sky determination). We regard these regions are the best approximation for the sky value.

The SDSS pipeline processing the data includes the sky subtraction; hence, the sky value for these frames is always about zero. For consistency, we did determine the sky value running мMм on the entire mosaic.

Photometric uncertainties introduced when determining the sky level are further discussed in Section 3.5.

\subsection{Removing foreground stars}

Contamination from foreground bulge or disc stars can be severe at low Galactic latitudes, and a number of methods have been proposed to tackle this issue. In their study on the integrated 2MASS photometry of Galactic GCs, Cohen et al. (2007) considered stars brighter than the tip of the RGB by $1.5 \mathrm{mag}$ as non-members and excluded them. However, it is difficult to use this approach close to the cluster centre (at least from the ground) because of crowding and the low spatial resolution of their (and our) data. Peng et al. (2006), for example, disregarded this correction.

Contaminating stars can be excluded in two ways: from their abnormal position in the cluster CMD, which implies that they are unlikely to be cluster members (e.g. if they lie well outside the cluster principal sequences), or from their measured proper motions, as cluster stars are unlikely to show detectable motions because of their great distances.

Although our data suffer from crowding, average seeing and poor spatial resolution, we were able to derive CMDs to identify likely foreground stars and clean the aperture magnitudes. We carried out stellar photometry with DAOPHOT and ALLSTAR (Stetson 1987, 1994). As a first step, up to 50 isolated and bright stars were selected to model a PSF, accounting for variation over the field by allowing quadratic variability. We used a PSF radius depending on the seeing: generally we used $4 \times$ FWHM but adopted a maximum of 15 pixels when the seeing was bad or the focus was mediocre. This aperture is large enough to remove the bulk of the contaminating star light but small enough to enable DAOPHOT to resolve the stars. DАОМАTCH and DAOMASTER were used to cross-match the different filters.

As a consequence of the low resolution of our data ( 0.396 arcsec pixels and a seeing between 1 and $\sim 2$ arcsec), crowding does obviously affect the final CMDs, which are not complete, especially close to the centre. However, these cover large fields, extending well beyond the half-light radius where crowding is not as important. A detailed analysis of these CMDs will be presented in a forthcoming paper.

As an example, Fig. 2 shows the CMD for NGC 5986: open circles represent stars within the half-light radius and dots denote stars from the entire field. This GC is located at a Galactic latitude of $b=13.27$, so some contamination from the disc may be expected, and is visible as a blue plume of stars above the turnoff. As mentioned above, we use the half-light radius to measure the aperture magnitudes of the cluster, so stars in this area (represented as open circles) that lie

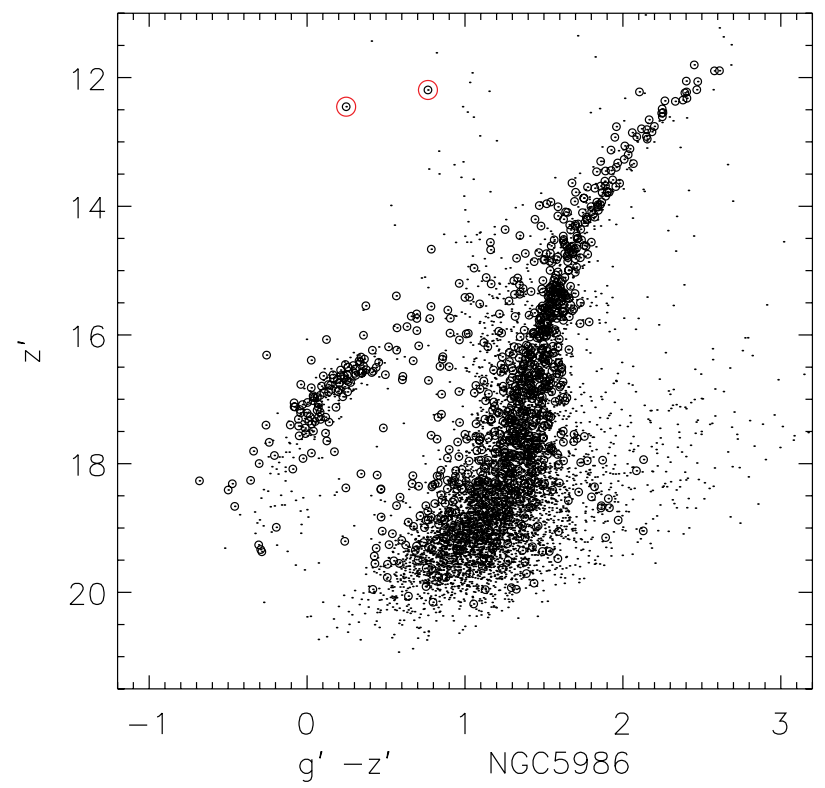

Figure 2. NGC 5986: open circles denote stars within $r_{\mathrm{h}}$; dots represent stars from the entire field. The two bright isolated stars in the CMD (indicated with red circles) are selected as candidate outliers and were checked for proper motions. See the text for more details.

outside of the principal sequences are possible contaminants. For confirmation, we checked the bright outliers, indicated by red circles in Fig. 2, for proper motions in the USNO-B1.0 catalogue (Monet et al. 2003) and the NOMAD catalogue (Zacharias et al. 2005), although in many cases these are not fully conclusive (e.g. see McDonald et al. 2013 for a similar approach to the bright asymptotic giant branch stars in NGC 4372). Once we are convinced that the star is a true non-member, the star is cleaned from the cluster photometry by subtracting its flux, based on the DAOPHOT PSF magnitudes, from the flux in the cluster aperture. Removing these stars in NGC 5986 results in magnitude corrections of $0.06(0.03,0.02,0.02)$ in $g^{\prime}\left(r^{\prime}\right.$, $i^{\prime}, z^{\prime}$, respectively). It is interesting to note that the contaminating stars in NGC 5986 would not have been removed if we had followed Cohen et al. (2007) as they are fainter than the RGB tip.

The magnitude corrections for foreground contamination may sometimes be very large, especially in poor clusters: for Pal 10 these corrections are $1.33(0.62,0.39,0.33) \mathrm{mag}$ in $g^{\prime}\left(r^{\prime}, i^{\prime}, z^{\prime}\right)$. This yields an $\sim 1$ mag correction for contamination in $g^{\prime}-z^{\prime}$.

A caveat is that differential reddening may shift foreground stars into the cluster principal sequences: this can be significant for clusters at low Galactic latitude, where extinction may be patchy (Alonso-García et al. 2012). While we discuss reddening-related issues extensively in our study on the colour-metallicity relation, specifically as these affect the colour-magnitude relation, we believe that a few such outliers will not significantly affect the derived colours.

For the CTIO data, an extract of the magnitudes and the applied contamination corrections (denoted as $\mathrm{CMD}_{g^{\prime}, r^{\prime}, i^{\prime}, z^{\prime}}$ ) is listed in Table 2. The complete table is available in the electronic version of this paper. Magnitudes and contamination corrections for the SDSS data are given in Table 3 .

\subsection{Comparison with previous work}

To test the reliability of our approach, we compare our $g^{\prime}$ and $z^{\prime}$ magnitudes with Peng et al. (2006), which also uses the half-light 
Table 2. Extract of the GC $g^{\prime} r^{\prime} i^{\prime} z^{\prime}$ magnitudes and errors based on CTIO observations. $\mathrm{CMD}_{g^{\prime}, r^{\prime}, i^{\prime}, z^{\prime}}$ presents the magnitude corrections based on the CMDs. The complete table is available in the online version of the paper.

\begin{tabular}{lcccccccccccc}
\hline ID & $g^{\prime}$ & $\sigma_{g^{\prime}}$ & $r^{\prime}$ & $\sigma_{r^{\prime}}$ & $i^{\prime}$ & $\sigma_{i^{\prime}}$ & $z^{\prime}$ & $\sigma_{z^{\prime}}$ & $\mathrm{CMD}_{g^{\prime}}$ & $\mathrm{CMD}_{r^{\prime}}$ & $\mathrm{CMD}_{i^{\prime}}$ & $\mathrm{CMD}_{z^{\prime}}$ \\
\hline NGC 104 & 4.912 & 0.030 & - & - & - & - & 3.677 & 0.044 & 0.00 & - & - & 0.00 \\
NGC 288 & 9.080 & 0.032 & 8.600 & 0.045 & 8.295 & 0.044 & 8.139 & 0.044 & 0.00 & 0.00 & 0.00 & 0.00 \\
NGC 362 & 7.471 & 0.030 & 6.925 & 0.043 & 6.618 & 0.043 & 6.419 & 0.043 & 0.01 & 0.02 & 0.02 & 0.03 \\
NGC 1261 & 9.474 & 0.031 & 8.995 & 0.045 & 8.744 & 0.045 & 8.582 & 0.045 & 0.03 & 0.05 & 0.06 & 0.06 \\
AM1 & 15.958 & 0.035 & 15.582 & 0.046 & 15.219 & 0.047 & 15.159 & 0.056 & 0.07 & 0.13 & 0.14 & 0.16 \\
NGC 1851 & 8.280 & 0.031 & 7.703 & 0.044 & 7.413 & 0.044 & 7.194 & 0.044 & 0.03 & 0.04 & 0.05 & 0.05 \\
NGC 1904 & 9.006 & 0.030 & 8.570 & 0.044 & 8.351 & 0.044 & 8.178 & 0.044 & 0.02 & 0.04 & 0.05 & 0.05 \\
NGC 2298 & 9.650 & 0.039 & 9.287 & 0.050 & 9.036 & 0.052 & 8.865 & 0.054 & 0.06 & 0.10 & 0.10 & 0.10 \\
NGC 2808 & 6.695 & 0.030 & 6.194 & 0.044 & 5.916 & 0.044 & 5.725 & 0.044 & 0.00 & 0.00 & 0.00 & 0.00 \\
\hline
\end{tabular}

Table 3. GC griz magnitudes and errors based on data from SDSS (Data Release 9). $\mathrm{CMD}_{g, r, i, z}$ presents the magnitude corrections based on the CMDs.

\begin{tabular}{lcccccccccccc}
\hline ID & $g$ & $\sigma_{g}$ & $r$ & $\sigma_{r}$ & $i$ & $\sigma_{i}$ & $z$ & $\sigma_{z}$ & $\mathrm{CMD}_{g}$ & $\mathrm{CMD}_{r}$ & $\mathrm{CMD}_{i}$ & $\mathrm{CMD}_{z}$ \\
\hline Whiting1 & 16.637 & 0.083 & 16.162 & 0.069 & 15.953 & 0.060 & 15.854 & 0.060 & 0.00 & 0.00 & 0.00 & 0.00 \\
Pal 1 & 15.118 & 0.042 & 14.769 & 0.033 & 14.615 & 0.030 & 14.522 & 0.032 & 0.46 & 0.62 & 0.67 & 0.69 \\
NGC 2419 & 11.198 & 0.009 & 10.727 & 0.009 & 10.473 & 0.009 & 10.371 & 0.010 & 0.01 & 0.01 & 0.01 & 0.01 \\
Pal 3 & 15.389 & 0.046 & 14.824 & 0.036 & 14.585 & 0.033 & 14.304 & 0.043 & 0.01 & 0.03 & 0.04 & 0.04 \\
Pal 4 & 15.284 & 0.052 & 14.636 & 0.058 & 14.293 & 0.083 & 14.074 & 0.107 & 0.00 & 0.00 & 0.00 & 0.00 \\
NGC 4147 & 11.185 & 0.010 & 10.823 & 0.008 & 10.670 & 0.007 & 10.443 & 0.005 & 0.00 & 0.00 & 0.00 & 0.00 \\
NGC 5024 & 8.518 & 0.003 & 8.122 & 0.004 & 7.991 & 0.003 & 7.676 & 0.003 & 0.00 & 0.00 & 0.00 & 0.00 \\
NGC 5053 & 10.734 & 0.012 & 10.379 & 0.008 & 10.164 & 0.007 & 10.062 & 0.005 & 0.00 & 0.00 & 0.00 & 0.00 \\
NGC 5272 & 7.071 & 0.002 & 6.712 & 0.002 & 6.555 & 0.001 & 6.135 & 0.002 & 0.00 & 0.00 & 0.00 & 0.00 \\
NGC 5466 & 10.180 & 0.013 & 9.725 & 0.011 & 9.466 & 0.009 & 9.413 & 0.009 & 0.00 & 0.00 & 0.00 & 0.00 \\
Pal 5 & 12.383 & 0.069 & 11.941 & 0.067 & 11.800 & 0.056 & 11.483 & 0.136 & 0.07 & 0.10 & 0.14 & 0.14 \\
Pal 14 & 14.654 & 0.028 & 13.937 & 0.047 & 13.689 & 0.044 & 13.641 & 0.052 & 1.02 & 0.58 & 0.79 & 0.81 \\
NGC 6205 & 6.903 & 0.005 & 6.667 & 0.003 & 6.397 & 0.004 & 6.076 & 0.002 & 0.00 & 0.00 & 0.00 & 0.00 \\
NGC 6229 & 10.419 & 0.007 & 9.876 & 0.005 & 9.751 & 0.005 & 9.489 & 0.004 & 0.00 & 0.00 & 0.00 & 0.00 \\
Pal 15 & 13.618 & 0.032 & 13.072 & 0.043 & 12.769 & 0.060 & 12.527 & 0.057 & 0.19 & 0.16 & 0.14 & 0.12 \\
NGC 6535 & 10.274 & 0.030 & 9.901 & 0.029 & 9.773 & 0.033 & 9.470 & 0.040 & 0.11 & 0.08 & 0.06 & 0.06 \\
NGC 6934 & 9.446 & 0.003 & 9.059 & 0.003 & 8.858 & 0.003 & 8.576 & 0.003 & 0.00 & 0.00 & 0.00 & 0.00 \\
NGC 7006 & 11.320 & 0.012 & 10.817 & 0.012 & 10.554 & 0.012 & 10.436 & 0.012 & 0.02 & 0.02 & 0.02 & 0.02 \\
NGC 7078 & 7.230 & 0.006 & 6.876 & 0.004 & 6.802 & 0.004 & 6.289 & 0.008 & 0.00 & 0.00 & 0.00 & 0.00 \\
NGC 7089 & 7.298 & 0.003 & 6.854 & 0.004 & 6.758 & 0.004 & 6.407 & 0.002 & 0.00 & 0.00 & 0.00 & 0.00 \\
Pal 13 & 15.736 & 0.060 & 15.392 & 0.050 & 15.270 & 0.048 & 15.147 & 0.049 & 0.00 & 0.00 & 0.00 & 0.00 \\
\hline
\end{tabular}

radius. Moreover, their Galactic GC data were based on the observations performed on 2004 June 5 and 6, so there is a considerable overlap with our sample. We match our apertures to theirs (some measurements of structural parameters have since changed) and we use the $E(B-V)$ values from Harris (1996) which were used by Peng et al. (2006) and a Cardelli, Clayton \& Mathis (1989) reddening law instead of the reddening values from Schlafly \& Finkbeiner (2011), for the sake of consistency in these comparisons. These are shown in Fig. 3: while there is no systematic offset, there are a couple of outliers, for which the magnitude difference with the earlier results (Peng et al. 2006) is larger than expected. It is unclear what the origin of the discrepancy is. In the next section, we discuss the origin of the photometric errors in more detail. It will become clear that the sky determination can strongly affect the final magnitudes, which we raise as possible cause for the variance when comparing to Peng et al. (2006). At least for NGC 5927, the observing log of the original Peng et al. (2006) hints at clouds or cirrus and this may be another possible reason for the difference. The rms scatter of our photometry, compared with Peng et al. (2006), is 0.08 (0.07, 0.09) for $g^{\prime}\left(z^{\prime}, g^{\prime}-z^{\prime}\right)$.

\subsection{Photometric errors}

Except for some poor clusters, the integrated magnitudes over the half-light radius have small random errors. The main contributions to the photometric error budget come from uncertainties in the photometric calibration and the centre determination (discussed above). For several clusters, we have $g^{\prime}$ and $z^{\prime}$ data available from consecutive short and long exposures or from observations obtained during different nights. In this case, the median magnitude of all observations is taken as the final magnitude in Table 2. In Fig. 4, we compare the magnitude differences between different observations (both performed on different nights or subsequent observations performed during the same night). The magnitudes in general compare well. However, there are some exceptions which will be treated later in this section.

First, we introduce some parameters that will be used to describe the details of these clusters. The standard way adopted in this study to estimate the sky level was described in Section 3.2. We now refer to this sky determination as method A. To test the influence of the sky determination on the cluster magnitude, we estimate the 

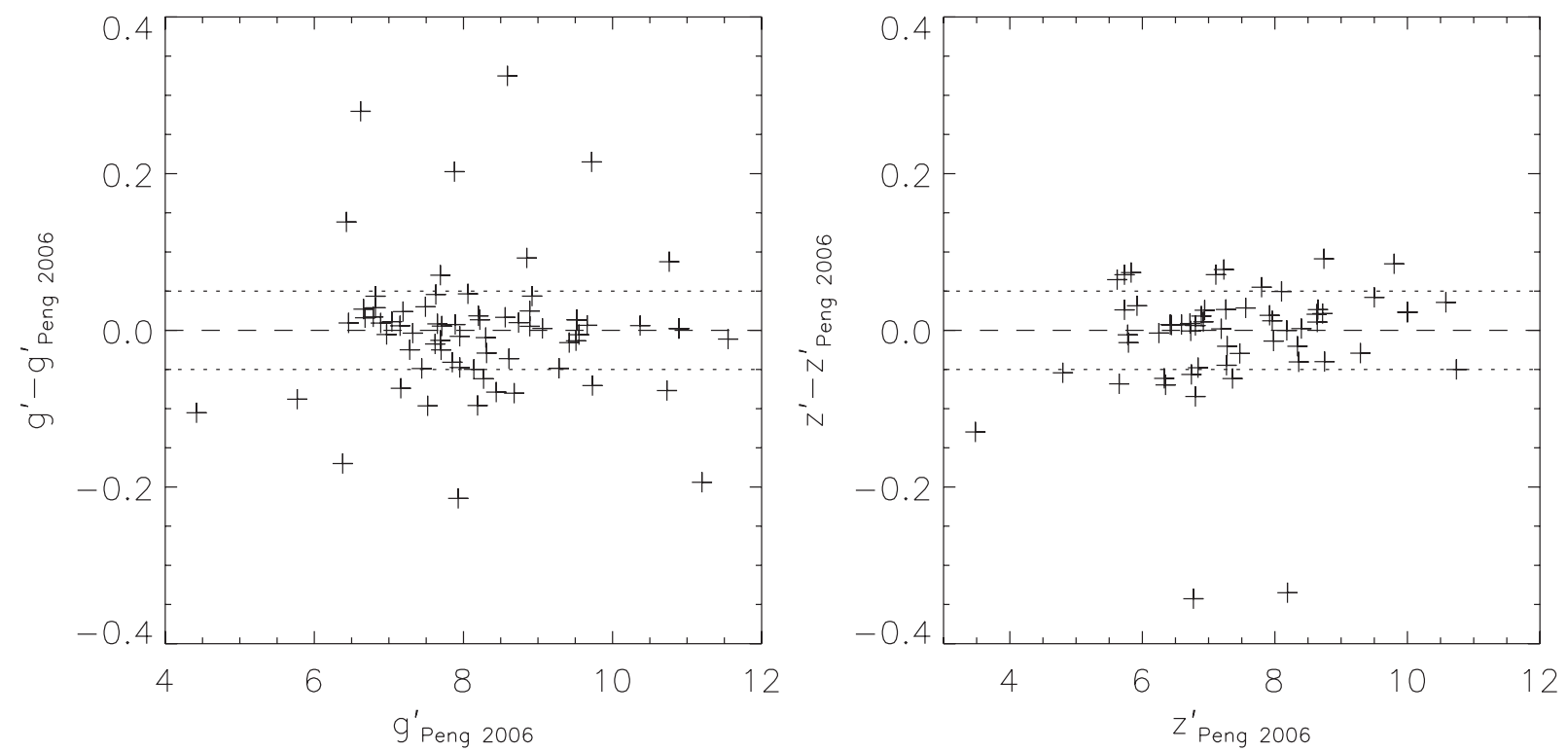

Figure 3. Comparison of the $g^{\prime}$ and $z^{\prime}$ magnitudes with the magnitudes obtained by Peng et al. (2006).
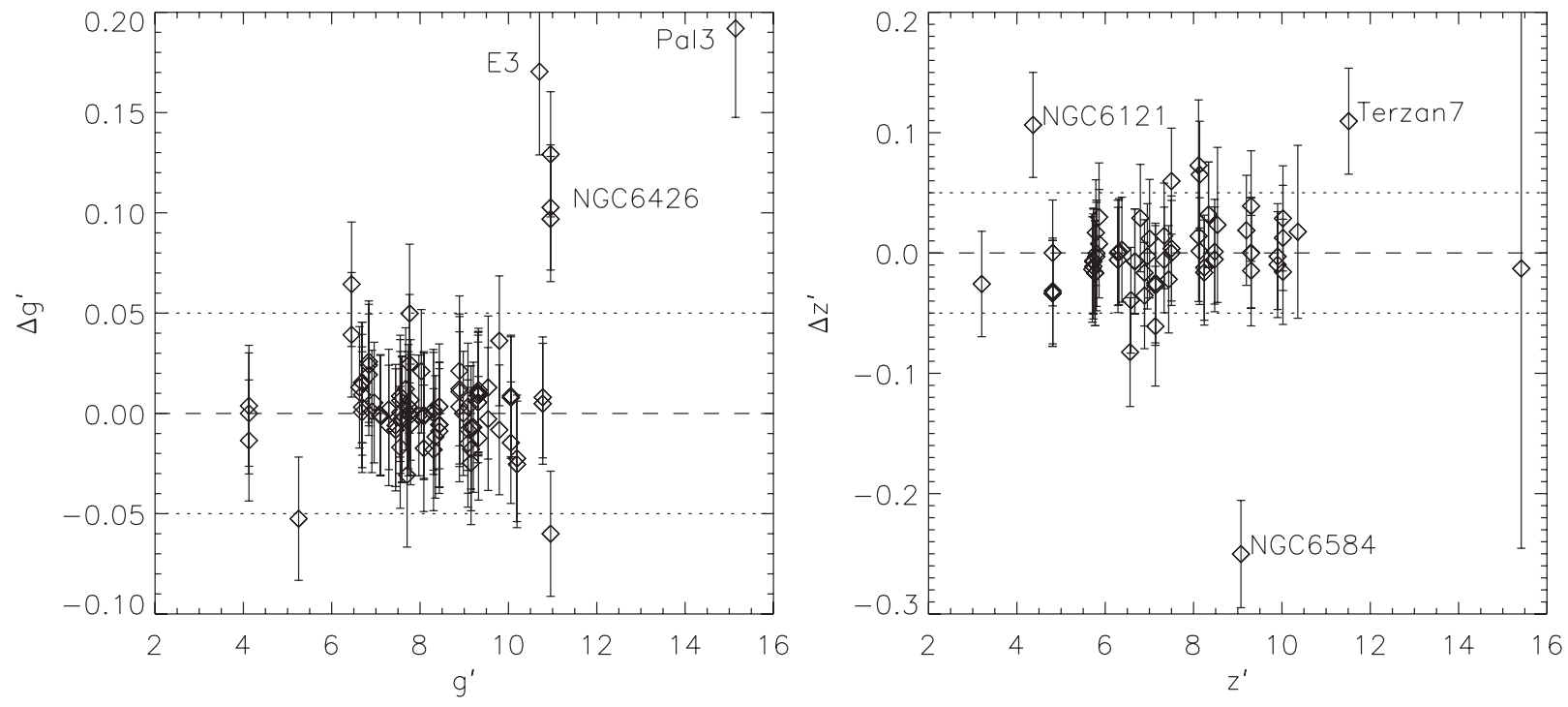

Figure 4. Comparison of $g^{\prime}$ and $z^{\prime}$ magnitudes, based on observations performed on different nights or performed subsequently during the same night. Error bars include the systematic error.

sky contribution using a sky band, centred in the cluster centre, with an inner radius of 900 pixels and a width of 100 pixels. We refer to the latter as method $\mathrm{B}$. The resulting magnitude difference between methods $\mathrm{A}$ and $\mathrm{B}$ is denoted as $\Delta_{\mathrm{AB}}^{*}$ (with '*' indicating the filter). Tests on SDSS data did not result in significantly different sky estimates using methods $\mathrm{A}$ and $\mathrm{B}$.

Another parameter used in the remainder of this section is $R_{\mathrm{GC} / \mathrm{Sky}, *}$, which is the ratio of the sky-subtracted cluster flux to the sky flux (both measured within a half-light radius). Hence, when $R_{\mathrm{GC} / \mathrm{Sky}, *}=1$, the sky contribution is as strong as the cluster contribution to the flux within $r_{\mathrm{h}} \cdot R_{\mathrm{GC} / \mathrm{Sky}, *}<1$ when the sky contribution is higher than the pure (sky-subtracted) cluster contribution.

E 3 is a faint old cluster (12.8 Gyr; Marín-Franch et al. 2009) that may have been truncated by tidal forces (van den Bergh, Demers \& Kunkel 1980). Observations of $60 \mathrm{~s}$ were performed on both 2004 June 5 and 2005 September 26, with magnitude differences between both nights of $0.17 \mathrm{mag}$ in $g^{\prime}$ and $0.02 \mathrm{mag}$ in $z^{\prime}$. It is suspicious that the magnitudes compare well for the $z^{\prime}$ filter, while they do not for the $g^{\prime}$ filter. In the latter filter, the average cluster surface brightness within a half-light radius is much lower than the brightness of the sky. Moreover, for the observations performed on 2004 June $5, R_{\mathrm{GC} / \mathrm{Sky}, g}=0.18$, while on 2005 September 26 $R_{\mathrm{GC} / \text { Sky }, g}=0.07$. This is a first indication that the magnitude difference could be attributable to the sky uncertainty. Using method $\mathrm{B}$ (with the sky radius) instead of method $\mathrm{A}$ (with the four corners) results in an absolute $g^{\prime}$ magnitude difference $\left|\Delta_{\mathrm{AB}}^{g^{\prime}}\right| \sim 0.1 \mathrm{mag}$, while the magnitude uncertainty is about $0.01 \mathrm{mag}$. This shows that the contribution of the sky uncertainty to the magnitude uncertainty is underestimated. We remark that the magnitude resulting using method B instead of method A was 0.1 mag brighter for the 2004 June 5 observation $\left(\Delta_{\mathrm{AB}}^{g^{\prime}} \sim 0.1 \mathrm{mag}\right)$, while it was $0.1 \mathrm{mag}$ fainter for the 2005 September 26 observation $\left(\Delta_{\mathrm{AB}}^{g^{\prime}} \sim-0.1 \mathrm{mag}\right)$. For the 
$z^{\prime}$ filter, $R_{\mathrm{GC} / \mathrm{Sky}, z}$ is about 0.02 for both nights. The $z^{\prime}$ magnitudes are differing for both nights by $\Delta_{\mathrm{AB}}^{z^{\prime}} \sim-0.1$ mag when using a sky band instead of the four-corner approach. Peng et al. (2006) did not include $\mathrm{E} 3$ in their analysis because of the significant reddening $(E(B-V) \sim 0.3$; Harris 1996).

NGC 6426, a high-reddening cluster with $E(B-V) \sim 0.36$, was observed on 2003 May 10 and on 2004 June 5 and 6 . The $g^{\prime}$-band magnitudes show significant variations between the nights: magnitudes obtained on 2003 May 10 were about 0.1 mag brighter than for the observations performed in 2004 June. Taking the median of the observations performed on the different nights resulted in a $g^{\prime}$ magnitude consistent with all the 2004 June observations. The resulting $z^{\prime}$ magnitudes are all within $0.04 \mathrm{mag}$. Again, we want to pinpoint the influence of the sky determination on the magnitude discrepancies. The ratio $R_{\mathrm{GC} / \mathrm{Sky}, g}$ is about 0.6 for the observations performed on 2003 May 10, while it is about 0.05 for the 2004 June 5 and 6 observations. $\Delta_{\mathrm{AB}}^{g^{\prime}} \lesssim 0.02 \mathrm{mag}$ for the 2003 May 10 observations, while these differences are as high as $0.34 \mathrm{mag}(0.24 \mathrm{mag})$ for the 2004 June 5 (6, respectively) observations. For the 75 and $410 \mathrm{~s} z^{\prime}$ observations taken on 2003 May 10, the ratio $R_{\mathrm{GC} / \mathrm{Sky}, z} \sim 0.1$, with $\Delta_{\mathrm{AB}}^{z^{\prime}}<0.03$. For the $60 \mathrm{~s}$ observation performed on 2004 June 5, $R_{\mathrm{GC} / \mathrm{Sky}, z} \sim 0.14$ and $\Delta_{\mathrm{AB}}^{z^{\prime}} \sim 0.24$, while for the 410 s observation taken on 2004 June $6, R_{\mathrm{GC} / \mathrm{Sky}, z} \sim 0.08$ and $\Delta_{\mathrm{AB}}^{z^{\prime}} \sim 0.15$. However, pure Poisson magnitude errors are smaller than 0.01. It is clear that some of the variation over the different nights can be attributed to the sky determination for this cluster.

Pal 3 is a low-reddening cluster $(E(B-V) \sim 0.04)$ and was observed on 2004 June 5 and 6 . The magnitude difference in $g^{\prime}$ was about $0.19 \mathrm{mag}$ (and $0.01 \mathrm{mag}$ in $z^{\prime}$ ). A bright star on the edge of the aperture complicates the determination of the magnitude. $R_{\mathrm{GC} / \mathrm{Sky}, g}$ amounts to about 0.05 on both nights, while $R_{\mathrm{GC} / \mathrm{Sky}, z}$ varies between 0.002 and 0.02 , so again the cluster is much fainter than the sky level. Using a sky band with ммм to estimate the sky value results in $g^{\prime}\left(z^{\prime}\right)$ magnitude differences up to $\Delta_{\mathrm{AB}}^{g^{\prime}} \sim 1 \mathrm{mag}$ ( $\Delta_{\mathrm{AB}}^{z^{\prime}} \sim 3.4 \mathrm{mag}$, respectively). The sky values obtained with ммм in the corners (as described in Section 3.2) and the sky band are consistent within the large uncertainties. However, the photometric magnitude uncertainties given by the routine are large (up to $\sigma_{z^{\prime}} \sim$ 0.23 ), but not as large as the differences invoked by different sky estimation methods.

NGC 6584 was observed on 2003 May 10 and 2004 June 5. While $g^{\prime}$ magnitudes compare well for both nights, $z^{\prime}$ magnitudes show larger differences. For the $z^{\prime}$ observations performed on June $5, R_{\mathrm{GC} / \mathrm{Sky}, z}$ is 0.86 , while for the observations taken on May 10 it amounts to 0.60 . $\Delta_{\mathrm{AB}}^{z^{\prime}} \sim 0.04 \mathrm{mag}$ for the 2004 June observation, while $\Delta_{\mathrm{AB}}^{z^{\prime}} \sim-0.02 \mathrm{mag}$ for the 2003 May observation. For this cluster, the sky determination seems not to be responsible for the magnitude offset. As a reference, we give some details on the $g^{\prime}$ observations as well. For the 37 and 270 s observations performed on 2003 May $10, R_{\mathrm{GC} / \mathrm{Sky}, g} \sim 6$ and $\Delta_{\mathrm{AB}}^{g^{\prime}}<0.01 \mathrm{mag}$. For the observation obtained on 2004 June $5, R_{\mathrm{GC} / \mathrm{Sky}, g} \sim 0.53$ and the magnitude difference (using a sky band instead of the corners) is about $\Delta_{\mathrm{AB}}^{g^{\prime}} \sim$ 0.03 mag.

Another cluster with a large difference between different observations is Terzan 7. This faint cluster was only observed on 2003 May 10, but consecutive $z^{\prime}$-band 79 and $410 \mathrm{~s}$ observations showed magnitude variations of about $0.1 \mathrm{mag}$. Because it is unlikely that, during a night that is considered photometric, the observing conditions change drastically in a $10 \mathrm{~min}$ timespan, this magnitude difference is surprising and deserves some special attention. This
Table 4. Median magnitude differences of clusters in common on different nights. Observations on 2005 September 26 had only few observations in common with other nights.

\begin{tabular}{lcc}
\hline \multicolumn{1}{c}{$\Delta$ Nights } & $g^{\prime}$ & $z^{\prime}$ \\
\hline $5 / 10 / 03-6 / 5 / 04$ & -0.004 & 0.015 \\
$5 / 10 / 03-6 / 6 / 04$ & -0.008 & 0.007 \\
$6 / 5 / 04-6 / 6 / 04$ & -0.002 & -0.002 \\
$6 / 5 / 04-9 / 26 / 05$ & -0.004 & 0.001 \\
\hline
\end{tabular}

cluster, at a Galactic latitude of $b \sim-20^{\circ}$, is associated with the Sagittarius stream, hence located in a crowded field. This obviously complicates the sky determination: there is a difference of 0.7 per cent between both sky determinations. However, as the surface brightness of the cluster is much lower than the sky level $\left(R_{\mathrm{GC} / \mathrm{Sky}, z} \sim 0.06\right)$, this sky level difference results in a magnitude difference as stated above. This example again stresses the difficulty and importance of obtaining a reliable sky value. Nevertheless, the resulting magnitude errors are lower than $0.01 \mathrm{mag}$; hence, the magnitude uncertainty is underestimated for this cluster. $\left|\Delta_{\mathrm{AB}}^{z^{\prime}}\right| \lesssim 0.01$ for both observations.

NGC 6121 is a bright high-reddening $(E(B-V) \sim 0.35)$ cluster showing a $z^{\prime}$ magnitude difference of $\sim 0.1$ mag between the short $1 \mathrm{~s}$ exposure obtained on 2003 May $10\left(z^{\prime} \sim 4.37\right)$ and the 11 s exposure performed on 2004 June $6\left(z^{\prime} \sim 4.47\right)$. This cluster is located in a very crowded field $\left(l \sim 351^{\circ}, b \sim 16^{\circ}\right)$, with a vast number of stars saturating the $60 \mathrm{~s}$ exposures. Nevertheless, for the short exposures, only few counts are collected to determine a reliable sky value. In the case of the $1 \mathrm{~s}$ exposure, the sky uncertainty obtained by MMM was larger than the sky value itself, with $R_{\mathrm{GC} / \mathrm{Sky}, z} \sim 1.2$ and $\Delta_{\mathrm{AB}}^{z^{\prime}} \sim-0.07 \mathrm{mag}$, while for the $11 \mathrm{~s}$ exposure, $R_{\mathrm{GC} / \mathrm{Sky}, z} \sim 1.2$ and $\Delta_{\mathrm{AB}}^{z^{\prime}} \lesssim 0.01 \mathrm{mag}$. On 2003 May 10, the short $1 \mathrm{~s}$ exposure was followed by a long $410 \mathrm{~s}$ exposure, which could obviously not be used to determine the aperture magnitude of the cluster because of saturation issues. However, when using the long exposure to determine a more reliable sky value and then applying this sky value to the $1 \mathrm{~s}$ exposure, we obtain a magnitude 0.05 mag fainter than the value obtained above. Note that this value is fully consistent with the median value of the magnitudes obtained on both 2003 May 10 and 2004 June 6.

The scatter in Fig. 4 is larger than we would expect given the known error budget (photometric, centroiding, etc.). We add a systematic contribution of $0.03 \mathrm{mag}$ for $g^{\prime}$ and $0.0435 \mathrm{mag}$ for $z^{\prime}$ ), for the remainder of this analysis, to reduce the derived $\chi^{2}$ to 1 and account for the additional photometric uncertainty. We cannot estimate this error for the other bands and we therefore adopt the $z^{\prime}$ error.

To demonstrate that this error is not caused by a systematic photometric shift of certain nights, we present in Table 4 the median differences for clusters in common for the given nights.

\subsection{Comparing CTIO and SDSS DR9}

The CTIO and SDSS subsamples have six clusters in common for the $g$ and $z$ bands and four GCs for $r$ and $i$ bands. In Fig. 5, we compare the different magnitudes for the different filters. The included error bars are the combined errors of both magnitudes. The rms values for the magnitude difference between CTIO and SDSS data are $0.10,0.18,0.21$ and 0.53 mag for the $g, r, i$ and 


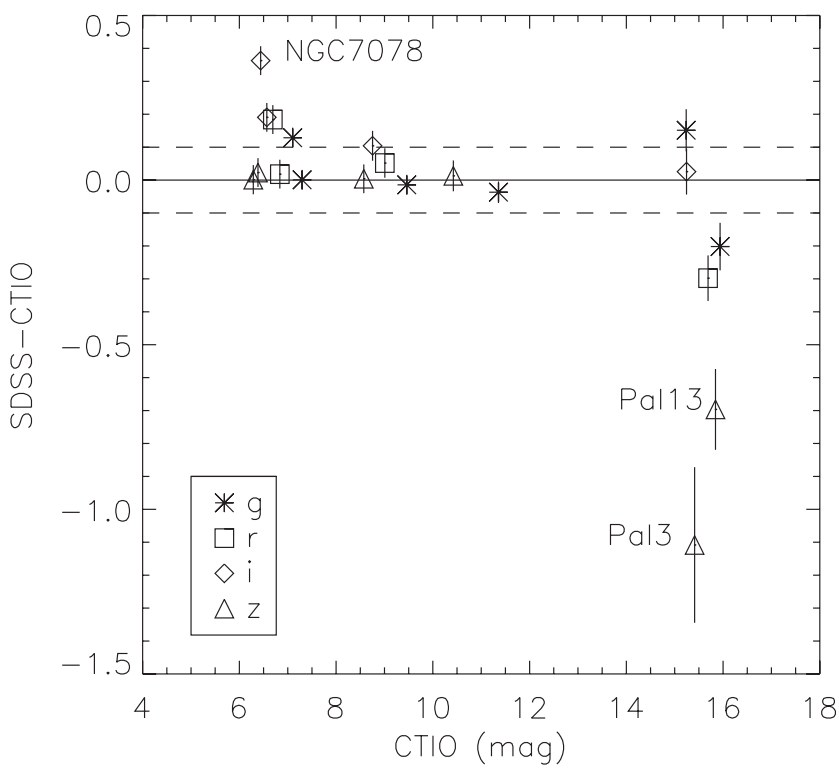

Figure 5. Comparison of magnitudes based on the CTIO observations and the SDSS DR9 survey. Error bars denote the combined errors on both magnitudes.

$z$ bands, respectively. NGC 7078 and NGC 7089 are the outliers in the $i$ band; Pal 3 and Pal 13 are the two $z$-band outliers. These objects contribute most to the high scatter.

Pal 3 has uncertain CTIO photometry, as was discussed in Section 3.5. It should be noted that the CTIO $g^{\prime}$ magnitude, based on the observations performed on 2004 June 6, is consistent with the SDSS magnitude (within the large photometric uncertainty for this faint cluster). However, $z^{\prime}$ magnitudes based on CTIO observations on both 2004 June 5 and 6 do not compare well with the SDSS magnitude for this filter. The issues regarding the sky determination for CTIO observations of this cluster were discussed in Section 3.5.

Pal 13 was only observed on 2005 September 26 and has one of the highest specific frequencies of blue stragglers in any known GC (Clark, Sandquist \& Bolte 2004). Based on the SDSS CMDs,

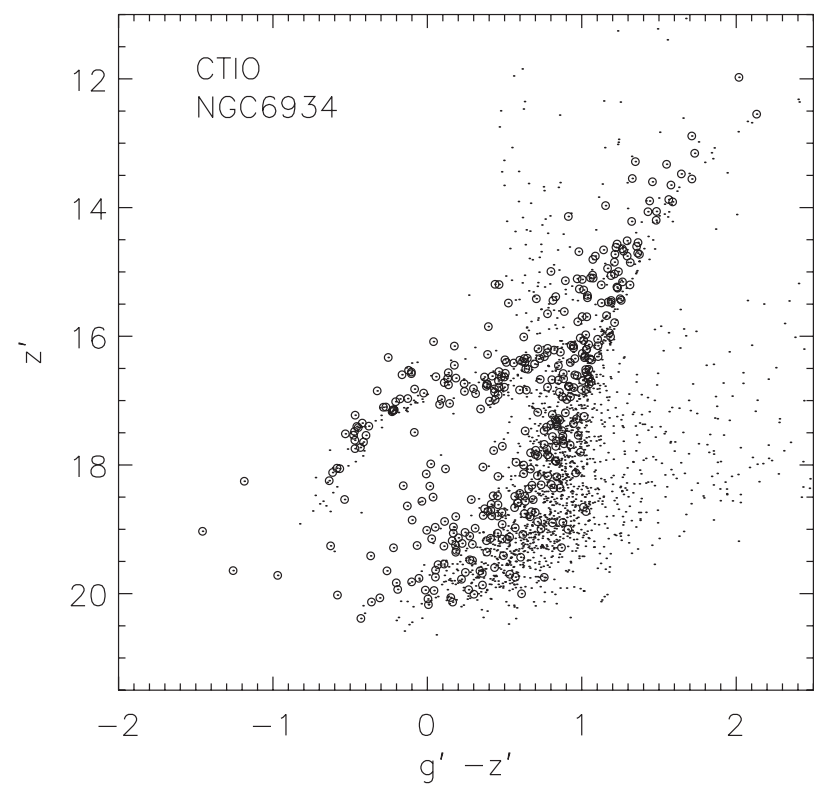

one candidate outlier was identified. However, the star did not have proper motions, and hence was not removed from the aperture photometry. This candidate outlier cannot explain the $g-z$ colour difference between SDSS and CTIO: removing the star would have resulted in a $g-z$ colour correction of -0.04 . In Section 3.5, it became clear that a small variation in the CTIO sky determination can result in a large magnitude difference, especially for faint clusters which have a lower surface brightness than the sky itself. Motivated by the latter argument, we reinspected the CTIO sky determination for Pal 13 . The $R_{\mathrm{GC} / \mathrm{Sky}, g} \sim 0.12$, while $R_{\mathrm{GC} / \mathrm{Sky}, z} \sim 0.01$, so the cluster flux contribution is much smaller than the sky contribution within a half-light radius. Using ммм on a sky ring of 900-1000 pixels (referred to as method B in Section 3.5) instead of the four-corner approach (method A) results in magnitude differences of $\Delta_{\mathrm{AB}}^{g^{\prime}} \lesssim 0.003$ mag, while these rise to $\Delta_{\mathrm{AB}}^{z^{\prime}} \sim 0.35 \mathrm{mag}$ in the $z^{\prime}$ band. The large photometric uncertainties are reflected in the magnitude errors, though these are smaller than the difference invoked by using the different sky estimation methods.

Fig. 6 presents CMDs for NGC 6934 based on CTIO and SDSS data. It is clear that the RGB in the SDSS CMD suffers from saturation (it is known that SDSS saturation starts at $r \sim 14$ ). Moreover, more blue stragglers are found in the CTIO CMD and the blue horizontal branch is more extended than in the SDSS CMD. Further analysis of the CMDs will be presented in a subsequent paper. Nevertheless, when comparing the integrated magnitudes of NGC 6934, the agreement is excellent in $g r z$ while in the $i$ band magnitudes show a larger difference.

The SDSS data for NGC 7078 are also saturated for the RGB stars. Only for the $z$ band the difference between SDSS and CTIO data is smaller than $0.01 \mathrm{mag}$; for the other filters the differences are much larger. We observed this cluster with the CTIO $0.9 \mathrm{~m}$ telescope on three different nights in the $g^{\prime}$ and $z^{\prime}$ filters. These three observations deviate less than $0.01 \mathrm{mag}$ from each other in both filters. The cluster was also observed with the $r^{\prime}$ and $i^{\prime}$ filters but only for one night.

For the future work, we use the CTIO-based magnitudes whenever GCs have magnitudes from both subsamples, except for Pal 3 and Pal 13, which are low-reddening clusters with very exceptional

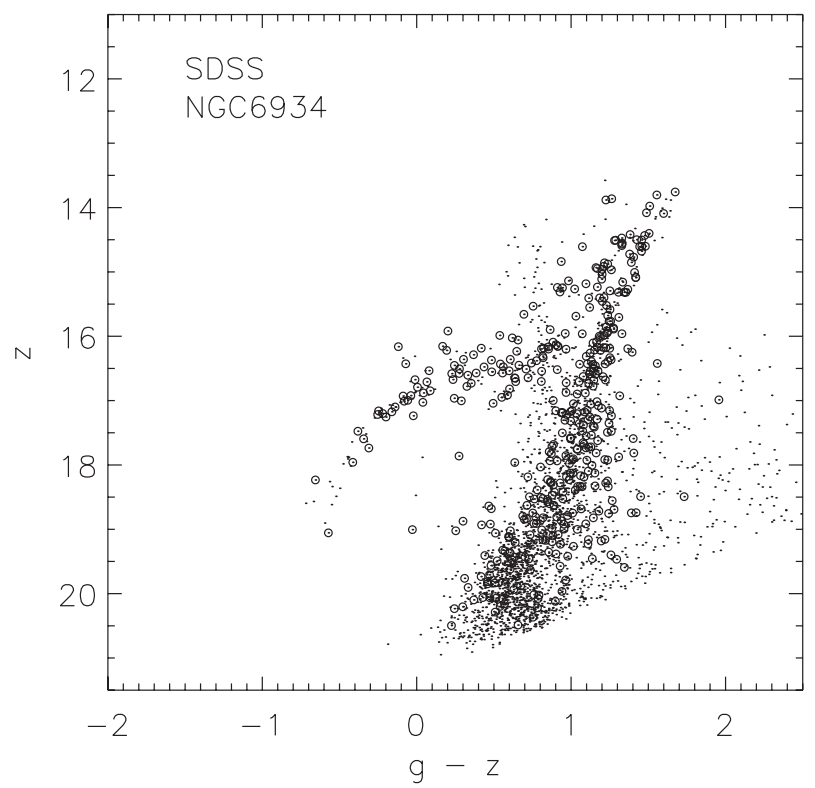

Figure 6. CMDs for NGC 6934 based on CTIO and SDSS data, using open circles for stars within $r_{\mathrm{h}}$ and dots for stars from the entire field. It is clear that saturation issues in the SDSS data sweep out the tip of the RGB. See the text for more details. 
CTIO colours $\left(g^{\prime}-z^{\prime} \sim 0\right)$, compared to $g^{\prime}-z^{\prime}$ colours of the other low-reddening clusters ranging between 0.4 and 1.4. We suspect that issues with the sky determination for the CTIO data are causing the offsets with the SDSS data for these faint clusters.

\section{SUMMARY}

In the current study, we presented integrated photometry for 96 Galactic GCs. We discuss a variety of issues, such as dealing with incomplete imaging (CCD cameras do not image the whole cluster), sky removal, calibration, the cleaning of contamination based on CMDs and proper motions and systematic errors. We obtained $g^{\prime}$ and $z^{\prime}$ magnitudes for about two-thirds of the Galactic GC system, making this the largest homogeneous optical sample based on the SDSS filter system. For about half of these clusters, we also present $r^{\prime}$ and $i^{\prime}$ photometry.

This work is the first of a series of papers, collected in the G2C2, exploiting this data set of SDSS photometry. Future studies will deal with the colour-metallicity relations, the CMDs, the spectral energy distributions, the structural parameters and the integrated spectroscopy.

\section{ACKNOWLEDGEMENTS}

We thankfully acknowledge the anonymous referee for very useful and thought-provoking comments. We would like to thank Giovanni Carraro for fruitful discussions. JV acknowledges the support of ESO through a studentship. JV and MB acknowledge the support of the Fund for Scientific Research Flanders (FWO-Vlaanderen). AJ acknowledges support by the Chilean Ministry for the Economy, Development, and Tourisms Programa Iniciativa Cientíca Milenio through grant P07-021-F, awarded to The Milky Way Millennium Nucleus.

The authors are grateful to CTIO for the hospitality and the dedicated assistance during the numerous observing runs.

This research has made use of NASA's Astrophysics Data System and the NED which is operated by the Jet Propulsion Laboratory, California Institute of Technology, under contract with the National Aeronautics and Space Administration.

This research also made use of Montage, funded by the National Aeronautics and Space Administration's Earth Science Technology Office, Computation Technologies Project, under Cooperative Agreement Number NCC5-626 between NASA and the California Institute of Technology. Montage is maintained by the NASA/IPAC Infrared Science Archive.

For this part of the research, we have made extensive use of the European Virtual Observatory applications ALADIN (Bonnarel et al. 2000) and тоРСАт (Taylor 2005). The Virtual Observatory is a project designed to provide the astronomical community with the data access and the research tools necessary to enable the exploration of the digital, multiwavelength Universe resident in the astronomical data archives. We used the applications provided by astrometry.net. ${ }^{3}$

\section{REFERENCES}

Ahn C. P. et al., 2012, ApJS, 203, 21

Alamo-Martínez K. A. et al., 2013, ApJ, 775, 20

Alonso-García J., Mateo M., Sen B., Banerjee M., Catelan M., Minniti D., von Braun K., 2012, AJ, 143, 70
Bellazzini M., 2007, A\&A, 473, 171

Bonnarel F. et al., 2000, A\&AS, 143, 33

Brodie J. P., Strader J., 2006, ARA\&A, 44, 193

Cardelli J. A., Clayton G. C., Mathis J. S., 1989, ApJ, 345, 245

Clark L. L., Sandquist E. L., Bolte M., 2004, AJ, 128, 3019

Cohen J. G., Hsieh S., Metchev S., Djorgovski S. G., Malkan M., 2007, AJ, 133, 99

Fukugita M., Ichikawa T., Gunn J. E., Doi M., Shimasaku K., Schneider D. P., 1996, AJ, 111, 1748

Goldsbury R., Richer H. B., Anderson J., Dotter A., Sarajedini A., Woodley K., 2010, AJ, 140, 1830

Harris W. E., 1991, ARA\&A, 29, 543

Harris W. E., 1996, AJ, 112, 1487

Jordán A. et al., 2005, ApJ, 634, 1002

Marín-Franch A. et al., 2009, ApJ, 694, 1498

McDonald I., Zijlstra A. A., Rajoelimanana A. F., Johnson C. I., 2013, MNRAS, 429, L65

McLaughlin D. E., van der Marel R. P., 2005, ApJS, 161, 304

Monet D. G. et al., 2003, AJ, 125, 984

Patat F., Carraro G., 2001, MNRAS, 325, 1591

Peacock M. B., Zepf S. E., Maccarone T. J., Kundu A., 2011, ApJ, 737, 5

Peng E. W. et al., 2006, ApJ, 639, 95

Schlafly E. F., Finkbeiner D. P., 2011, ApJ, 737, 103

Sinnott B., Hou A., Anderson R., Harris W. E., Woodley K. A., 2010, AJ, 140,2101

Smith J. A. et al., 2000, BAAS, 32, 1424

Smith J. A. et al., 2002, AJ, 123, 2121

Stetson P. B., 1987, PASP, 99, 191

Stetson P. B., 1994, PASP, 106, 250

Taylor M. B., 2005, in Shopbell P., Britton M., Ebert R., eds, ASP Conf. Ser. Vol. 347, Astronomical Data Analysis Software and Systems XIV. Astron. Soc. Pac., San Francisco, p. 29

Trager S. C., Djorgovski S., King I. R., 1993, in Djorgovski S. G., Meylan G., eds, ASP Conf. Ser. Vol. 50, Structure and Dynamics of Globular Clusters. Astron. Soc. Pac., San Francisco, p. 347

Trager S. C., King I. R., Djorgovski S., 1995, AJ, 109, 218

van den Bergh S., Demers S., Kunkel W. E., 1980, ApJ, 239, 112

van Dokkum P. G., 2001, PASP, 113, 1420

Vanderbeke J. et al., 2014, MNRAS, 437, 1734 (Paper II)

Vickers J. J., Grebel E. K., Huxor A. P., 2012, AJ, 143, 86

West M. J., Côté P., Marzke R. O., Jordán A., 2004, Nat, 427, 31

York D. G. et al., 2000, AJ, 120, 1579

Zacharias N., Monet D. G., Levine S. E., Urban S. E., Gaume R., Wycoff G. L., 2005, VizieR Online Data Catalog, 1297, 0

\section{SUPPORTING INFORMATION}

Additional Supporting Information may be found in the online version of this article:

Table 2. Extract of the GC $g^{\prime} r^{\prime} i^{\prime} z^{\prime}$ magnitudes and errors based on CTIO observations (http://mnras.oxfordjournals.org/ lookup/suppl/doi:10.1093/mnras/stt2002/-/ DC1)

Please note: Oxford University Press are not responsible for the content or functionality of any supporting materials supplied by the authors. Any queries (other than missing material) should be directed to the corresponding author for the article.

\footnotetext{
${ }^{3}$ http://astrometry.net/
} 
Table 2: GC $g^{\prime} r^{\prime} i^{\prime} z^{\prime}$ magnitudes and errors.

\begin{tabular}{|c|c|c|c|c|c|c|c|c|c|c|c|c|}
\hline ID & $g^{\prime}$ & $\sigma_{g^{\prime}}$ & $r^{\prime}$ & $\sigma_{r^{\prime}}$ & $i^{\prime}$ & $\sigma_{i^{\prime}}$ & $z^{\prime}$ & $\sigma_{z^{\prime}}$ & $\mathrm{CMD}_{g^{\prime}}$ & $\mathrm{CMD}_{r^{\prime}}$ & $\mathrm{CMD}_{i^{\prime}}$ & $\mathrm{CMD}_{z^{\prime}}$ \\
\hline NGC104 & 4.912 & 0.030 & $\ldots$ & $\ldots$ & $\ldots$ & $\ldots$ & 3.677 & 0.044 & 0.00 & $\ldots$ & $\ldots$ & 0.00 \\
\hline NGC288 & 9.080 & 0.032 & 8.600 & 0.045 & 8.295 & 0.044 & 8.139 & 0.044 & 0.00 & 0.00 & 0.00 & 0.00 \\
\hline NGC362 & 7.471 & 0.030 & 6.925 & 0.043 & 6.618 & 0.043 & 6.419 & 0.043 & 0.01 & 0.02 & 0.02 & 0.03 \\
\hline NGC1261 & 9.474 & 0.031 & 8.995 & 0.045 & 8.744 & 0.045 & 8.582 & 0.045 & 0.03 & 0.05 & 0.06 & 0.06 \\
\hline AM1 & 15.958 & 0.035 & 15.582 & 0.046 & 15.219 & 0.047 & 15.159 & 0.056 & 0.07 & 0.13 & 0.14 & 0.16 \\
\hline NGC1851 & 8.280 & 0.031 & 7.703 & 0.044 & 7.413 & 0.044 & 7.194 & 0.044 & 0.03 & 0.04 & 0.05 & 0.05 \\
\hline NGC1904 & 9.006 & 0.030 & 8.570 & 0.044 & 8.351 & 0.044 & 8.178 & 0.044 & 0.02 & 0.04 & 0.05 & 0.05 \\
\hline NGC2298 & 9.650 & 0.039 & 9.287 & 0.050 & 9.036 & 0.052 & 8.865 & 0.054 & 0.06 & 0.10 & 0.10 & 0.10 \\
\hline NGC2808 & 6.695 & 0.030 & 6.194 & 0.044 & 5.916 & 0.044 & 5.725 & 0.044 & 0.00 & 0.00 & 0.00 & 0.00 \\
\hline E3 & 10.787 & 0.041 & $\ldots$ & $\ldots$ & $\ldots$ & $\ldots$ & 10.368 & 0.072 & 0.00 & $\ldots$ & $\ldots$ & 0.00 \\
\hline Pal3 & 15.238 & 0.044 & $\ldots$ & $\ldots$ & $\ldots$ & $\ldots$ & 15.412 & 0.232 & 0.00 & $\ldots$ & $\ldots$ & 0.00 \\
\hline NGC3201 & 7.006 & 0.033 & $\ldots$ & $\ldots$ & $\ldots$ & $\ldots$ & $\ldots$ & $\ldots$ & 0.01 & $\ldots$ & $\ldots$ & 0.00 \\
\hline NGC4372 & 6.489 & 0.031 & $\ldots$ & $\ldots$ & $\ldots$ & $\ldots$ & 5.796 & 0.044 & 0.00 & $\ldots$ & $\ldots$ & 0.00 \\
\hline Rup106 & 11.981 & 0.031 & $\ldots$ & $\ldots$ & $\ldots$ & $\ldots$ & 11.204 & 0.168 & 0.59 & $\ldots$ & $\ldots$ & 0.69 \\
\hline NGC4590 & 8.903 & 0.037 & $\ldots$ & $\ldots$ & $\ldots$ & $\ldots$ & 8.127 & 0.054 & 0.00 & $\ldots$ & $\ldots$ & 0.00 \\
\hline NGC4833 & 7.034 & 0.034 & $\ldots$ & $\ldots$ & $\ldots$ & $\ldots$ & $\ldots$ & $\ldots$ & 0.03 & $\ldots$ & $\ldots$ & 0.02 \\
\hline NGC5139 & 4.131 & 0.030 & $\ldots$ & $\ldots$ & $\ldots$ & $\ldots$ & 3.198 & 0.044 & 0.01 & $\ldots$ & $\ldots$ & 0.01 \\
\hline NGC5286 & 7.551 & 0.030 & $\ldots$ & $\ldots$ & $\ldots$ & $\ldots$ & 6.664 & 0.044 & 0.00 & $\ldots$ & $\ldots$ & 0.00 \\
\hline NGC5634 & 10.060 & 0.030 & $\ldots$ & $\ldots$ & $\ldots$ & $\ldots$ & 9.205 & 0.046 & 0.00 & $\ldots$ & $\ldots$ & 0.00 \\
\hline NGC5694 & 10.781 & 0.030 & $\ldots$ & $\ldots$ & $\ldots$ & $\ldots$ & 9.899 & 0.044 & 0.00 & $\ldots$ & $\ldots$ & 0.00 \\
\hline IC4499 & 10.256 & 0.032 & 9.820 & 0.044 & 9.520 & 0.044 & 9.372 & 0.044 & 0.18 & 0.24 & 0.23 & 0.22 \\
\hline NGC5824 & 9.321 & 0.030 & 8.876 & 0.043 & 8.648 & 0.043 & 8.477 & 0.043 & 0.00 & 0.00 & 0.00 & 0.00 \\
\hline NGC5897 & 9.171 & 0.031 & 8.835 & 0.044 & 8.542 & 0.044 & 8.414 & 0.044 & 0.03 & 0.04 & 0.04 & 0.04 \\
\hline NGC5904 & 6.861 & 0.030 & $\ldots$ & $\ldots$ & $\ldots$ & $\ldots$ & 5.867 & 0.045 & 0.00 & $\ldots$ & $\ldots$ & 0.00 \\
\hline NGC5927 & 7.785 & 0.030 & 7.184 & 0.043 & 6.816 & 0.044 & 6.521 & 0.045 & 0.00 & 0.00 & 0.00 & 0.00 \\
\hline NGC5946 & 8.340 & 0.031 & 7.889 & 0.044 & 7.645 & 0.045 & 7.477 & 0.045 & 0.30 & 0.14 & 0.08 & 0.05 \\
\hline NGC5986 & 7.794 & 0.031 & 7.349 & 0.045 & 7.072 & 0.044 & 6.894 & 0.044 & 0.06 & 0.03 & 0.02 & 0.02 \\
\hline NGC6093 & 7.924 & 0.030 & 7.417 & 0.044 & 7.148 & 0.044 & 6.939 & 0.044 & 0.00 & 0.00 & 0.00 & 0.00 \\
\hline NGC6121 & 5.251 & 0.031 & $\ldots$ & $\ldots$ & $\ldots$ & $\ldots$ & 4.435 & 0.044 & 0.03 & $\ldots$ & $\ldots$ & 0.02 \\
\hline NGC6101 & 10.171 & 0.032 & 9.736 & 0.045 & 9.460 & 0.044 & 9.301 & 0.046 & 0.00 & 0.00 & 0.00 & 0.00 \\
\hline NGC6144 & 7.876 & 0.031 & 7.811 & 0.044 & 7.689 & 0.044 & 7.602 & 0.044 & 0.19 & 0.13 & 0.09 & 0.07 \\
\hline NGC6139 & 7.375 & 0.030 & $\ldots$ & $\ldots$ & $\ldots$ & $\ldots$ & 6.623 & 0.044 & 0.00 & $\ldots$ & $\ldots$ & 0.00 \\
\hline NGC6171 & 8.084 & 0.032 & $\ldots$ & $\ldots$ & $\ldots$ & $\ldots$ & 7.109 & 0.050 & 0.00 & $\ldots$ & $\ldots$ & 0.00 \\
\hline NGC6218 & 7.691 & 0.036 & $\ldots$ & $\ldots$ & $\ldots$ & $\ldots$ & 6.653 & 0.053 & 0.00 & $\ldots$ & $\ldots$ & 0.00 \\
\hline NGC6235 & 9.542 & 0.036 & $\ldots$ & $\ldots$ & $\ldots$ & $\ldots$ & 8.552 & 0.064 & 0.00 & $\ldots$ & $\ldots$ & 0.00 \\
\hline NGC6254 & 6.842 & 0.031 & $\ldots$ & $\ldots$ & $\ldots$ & $\ldots$ & $\ldots$ & $\ldots$ & $\ldots$ & $\ldots$ & $\ldots$ & $\ldots$ \\
\hline NGC6273 & 6.909 & 0.031 & $\ldots$ & $\ldots$ & $\ldots$ & $\ldots$ & 5.801 & 0.044 & 0.00 & $\ldots$ & $\ldots$ & 0.00 \\
\hline NGC6284 & 9.247 & 0.030 & $\ldots$ & $\ldots$ & $\ldots$ & $\ldots$ & 8.145 & 0.044 & 0.00 & $\ldots$ & $\ldots$ & 0.00 \\
\hline NGC6287 & 8.121 & 0.030 & 7.811 & 0.046 & 7.580 & 0.045 & 7.426 & 0.044 & 0.00 & 0.00 & 0.00 & 0.00 \\
\hline NGC6293 & 7.440 & 0.030 & $\ldots$ & $\ldots$ & $\ldots$ & $\ldots$ & 6.999 & 0.044 & 0.00 & $\ldots$ & $\ldots$ & 0.05 \\
\hline NGC6304 & 7.677 & 0.030 & $\ldots$ & $\ldots$ & $\ldots$ & $\ldots$ & 6.313 & 0.044 & 0.00 & $\ldots$ & $\ldots$ & 0.00 \\
\hline NGC6316 & 8.131 & 0.030 & $\ldots$ & $\ldots$ & $\ldots$ & $\ldots$ & 7.090 & 0.043 & 0.00 & $\ldots$ & $\ldots$ & 0.00 \\
\hline NGC6333 & 7.467 & 0.032 & $\ldots$ & $\ldots$ & $\ldots$ & $\ldots$ & 6.676 & 0.044 & 0.00 & $\ldots$ & $\ldots$ & 0.00 \\
\hline NGC6342 & 9.327 & 0.031 & $\ldots$ & $\ldots$ & $\ldots$ & $\ldots$ & 8.231 & 0.044 & 0.00 & $\ldots$ & $\ldots$ & 0.00 \\
\hline NGC6356 & 8.499 & 0.031 & $\ldots$ & $\ldots$ & $\ldots$ & $\ldots$ & 7.231 & 0.048 & 0.01 & $\ldots$ & $\ldots$ & 0.03 \\
\hline NGC6355 & 7.413 & 0.039 & $\ldots$ & $\ldots$ & $\ldots$ & $\ldots$ & 6.908 & 0.049 & 0.00 & $\ldots$ & $\ldots$ & 0.00 \\
\hline NGC6352 & 7.788 & 0.035 & $\ldots$ & $\ldots$ & $\ldots$ & $\ldots$ & 6.562 & 0.044 & 0.00 & $\ldots$ & $\ldots$ & 0.00 \\
\hline IC1257 & $\ldots$ & $\ldots$ & 10.927 & 0.045 & 10.719 & 0.046 & $\ldots$ & $\ldots$ & $\ldots$ & 0.12 & 0.13 & $\ldots$ \\
\hline NGC6366 & 7.736 & 0.031 & $\ldots$ & $\ldots$ & $\ldots$ & $\ldots$ & 6.769 & 0.044 & 0.23 & $\ldots$ & $\ldots$ & 0.10 \\
\hline NGC6362 & 8.429 & 0.031 & $\ldots$ & $\ldots$ & $\ldots$ & $\ldots$ & 7.334 & 0.044 & 0.00 & $\ldots$ & $\ldots$ & 0.00 \\
\hline NGC6388 & 6.962 & 0.030 & $\ldots$ & $\ldots$ & $\ldots$ & $\ldots$ & 5.754 & 0.044 & 0.00 & $\ldots$ & $\ldots$ & 0.00 \\
\hline NGC6402 & 7.503 & 0.030 & $\ldots$ & $\ldots$ & $\ldots$ & $\ldots$ & 6.203 & 0.044 & 0.00 & $\ldots$ & $\ldots$ & 0.00 \\
\hline NGC6401 & 7.373 & 0.036 & $\ldots$ & $\ldots$ & $\ldots$ & $\ldots$ & $\ldots$ & $\ldots$ & $\ldots$ & $\ldots$ & $\ldots$ & $\ldots$ \\
\hline NGC6397 & $\ldots$ & $\ldots$ & $\ldots$ & $\ldots$ & $\ldots$ & $\ldots$ & 5.450 & 0.044 & 0.00 & $\ldots$ & $\ldots$ & 0.00 \\
\hline NGC6426 & 11.051 & 0.031 & $\ldots$ & $\ldots$ & $\ldots$ & $\ldots$ & 10.030 & 0.044 & 0.00 & $\ldots$ & $\ldots$ & 0.00 \\
\hline NGC6440 & 7.504 & 0.030 & $\ldots$ & $\ldots$ & $\ldots$ & $\ldots$ & 6.080 & 0.044 & 0.00 & $\ldots$ & $\ldots$ & 0.00 \\
\hline NGC6441 & 6.635 & 0.030 & $\ldots$ & $\ldots$ & $\ldots$ & $\ldots$ & 5.700 & 0.044 & 0.00 & $\ldots$ & $\ldots$ & 0.00 \\
\hline
\end{tabular}


Table 2 - continued from previous page

\begin{tabular}{|c|c|c|c|c|c|c|c|c|c|c|c|c|}
\hline ID & $g^{\prime}$ & $\sigma_{g^{\prime}}$ & $r^{\prime}$ & $\sigma_{r^{\prime}}$ & $i^{\prime}$ & $\sigma_{i^{\prime}}$ & $z^{\prime}$ & $\sigma_{z^{\prime}}$ & $\mathrm{CMD}_{g^{\prime}}$ & $\mathrm{CMD}_{r^{\prime}}$ & $\mathrm{CMD}_{i^{\prime}}$ & $\mathrm{CMD}_{z^{\prime}}$ \\
\hline NGC6453 & 9.016 & 0.031 & 8.381 & 0.044 & $\ldots$ & $\cdots$ & $\ldots$ & $\cdots$ & & $\ldots$ & $\ldots$ & 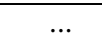 \\
\hline NGC6496 & 10.095 & 0.055 & $\ldots$ & $\ldots$ & $\ldots$ & $\ldots$ & $\ldots$ & $\ldots$ & 0.49 & $\ldots$ & $\ldots$ & 0.55 \\
\hline NGC6517 & 8.298 & 0.030 & 7.918 & 0.043 & 7.613 & 0.044 & 7.411 & 0.044 & 0.00 & 0.00 & 0.00 & 0.00 \\
\hline NGC6539 & 7.740 & 0.036 & 7.148 & 0.044 & 6.738 & 0.044 & 6.457 & 0.045 & 0.22 & 0.14 & 0.12 & 0.11 \\
\hline NGC6544 & 3.941 & 0.033 & 4.341 & 0.045 & $\ldots$ & $\ldots$ & $\ldots$ & $\ldots$ & $\ldots$ & $\ldots$ & $\ldots$ & $\ldots$ \\
\hline NGC6541 & 7.262 & 0.031 & $\ldots$ & $\ldots$ & $\ldots$ & $\ldots$ & $\ldots$ & $\ldots$ & 0.00 & $\ldots$ & $\ldots$ & 0.01 \\
\hline NGC6553 & 5.254 & 0.030 & 5.145 & 0.044 & 4.966 & 0.044 & 4.822 & 0.044 & 0.19 & 0.09 & 0.06 & 0.04 \\
\hline NGC6558 & 7.751 & 0.035 & 7.238 & 0.045 & 6.976 & 0.045 & 6.866 & 0.044 & 0.00 & 0.00 & 0.00 & 0.00 \\
\hline IC1276 & 6.814 & 0.037 & 6.427 & 0.044 & 6.148 & 0.044 & 6.016 & 0.045 & 0.19 & 0.14 & 0.18 & 0.22 \\
\hline NGC6569 & 8.801 & 0.031 & 8.048 & 0.044 & 7.605 & 0.044 & 7.278 & 0.044 & 0.05 & 0.04 & 0.03 & 0.04 \\
\hline NGC6584 & 9.793 & 0.032 & 9.279 & 0.051 & 9.017 & 0.048 & 8.947 & 0.045 & 0.00 & 0.00 & 0.00 & 0.00 \\
\hline NGC6624 & 8.341 & 0.031 & 7.696 & 0.044 & 7.285 & 0.046 & 7.009 & 0.049 & 0.00 & 0.00 & 0.00 & 0.00 \\
\hline NGC6626 & 6.180 & 0.030 & 5.706 & 0.044 & 5.424 & 0.044 & $\ldots$ & $\ldots$ & $\ldots$ & $\ldots$ & $\ldots$ & $\ldots$ \\
\hline NGC6638 & 8.977 & 0.031 & 8.351 & 0.044 & 8.033 & 0.044 & 7.849 & 0.044 & 0.00 & 0.00 & 0.00 & 0.00 \\
\hline NGC6637 & 8.295 & 0.064 & 7.693 & 0.048 & 7.306 & 0.049 & $\ldots$ & $\ldots$ & 0.00 & 0.00 & 0.00 & 0.00 \\
\hline NGC6642 & 9.309 & 0.032 & $\ldots$ & $\ldots$ & $\ldots$ & $\ldots$ & 8.152 & 0.046 & 0.11 & $\ldots$ & $\ldots$ & 0.12 \\
\hline NGC6652 & 9.818 & 0.031 & $\ldots$ & $\ldots$ & $\ldots$ & $\ldots$ & 8.737 & 0.046 & 0.00 & $\ldots$ & $\ldots$ & 0.00 \\
\hline Pal8 & 10.569 & 0.031 & $\ldots$ & $\ldots$ & $\ldots$ & $\ldots$ & 9.349 & 0.045 & 0.01 & $\ldots$ & $\ldots$ & 0.11 \\
\hline NGC6681 & 8.859 & 0.030 & $\ldots$ & $\ldots$ & $\ldots$ & $\ldots$ & $\ldots$ & $\ldots$ & 0.00 & $\ldots$ & $\ldots$ & 0.00 \\
\hline NGC6712 & 7.955 & 0.030 & $\ldots$ & $\ldots$ & $\ldots$ & $\ldots$ & $\ldots$ & $\ldots$ & 0.00 & $\ldots$ & $\ldots$ & 0.00 \\
\hline NGC6715 & 7.973 & 0.030 & $\ldots$ & $\ldots$ & $\ldots$ & $\ldots$ & 6.946 & 0.043 & 0.00 & $\ldots$ & $\ldots$ & 0.00 \\
\hline NGC6723 & 7.585 & 0.031 & $\ldots$ & $\ldots$ & $\ldots$ & $\ldots$ & 6.810 & 0.045 & 0.00 & $\ldots$ & $\ldots$ & 0.00 \\
\hline NGC6749 & 6.735 & 0.045 & $\ldots$ & $\ldots$ & $\ldots$ & $\ldots$ & 6.341 & 0.044 & 0.41 & $\ldots$ & $\ldots$ & 0.05 \\
\hline NGC6760 & 8.304 & 0.030 & $\ldots$ & $\ldots$ & $\ldots$ & $\ldots$ & 6.522 & 0.044 & 0.00 & $\ldots$ & $\ldots$ & 0.00 \\
\hline NGC6779 & 8.613 & 0.030 & 8.138 & 0.044 & 7.879 & 0.044 & 7.748 & 0.044 & 0.00 & 0.00 & 0.00 & 0.00 \\
\hline Terzan7 & 12.469 & 0.031 & $\ldots$ & $\ldots$ & $\ldots$ & $\ldots$ & 11.567 & 0.044 & 0.00 & $\ldots$ & $\ldots$ & 0.00 \\
\hline Pal10 & 7.862 & 0.039 & 7.498 & 0.050 & 7.328 & 0.056 & 7.216 & 0.047 & 1.33 & 0.62 & 0.39 & 0.33 \\
\hline Pal11 & 11.439 & 0.034 & $\ldots$ & $\ldots$ & $\ldots$ & $\ldots$ & 10.413 & 0.048 & 0.00 & $\ldots$ & $\ldots$ & 0.00 \\
\hline NGC6838 & 7.973 & 0.039 & $\ldots$ & $\ldots$ & $\ldots$ & $\ldots$ & $\ldots$ & $\ldots$ & 0.00 & $\ldots$ & $\ldots$ & 0.00 \\
\hline NGC6864 & 9.167 & 0.030 & 8.608 & 0.044 & 8.320 & 0.044 & 8.099 & 0.044 & 0.00 & 0.00 & 0.00 & 0.00 \\
\hline NGC6934 & 9.460 & 0.030 & 9.007 & 0.045 & 8.754 & 0.045 & 8.572 & 0.044 & 0.00 & 0.00 & 0.00 & 0.00 \\
\hline NGC6981 & 10.132 & 0.031 & 9.666 & 0.045 & 9.391 & 0.045 & 9.213 & 0.046 & 0.00 & 0.00 & 0.00 & 0.00 \\
\hline NGC7006 & 11.357 & 0.030 & $\ldots$ & $\ldots$ & $\ldots$ & $\ldots$ & 10.423 & 0.045 & 0.03 & $\ldots$ & $\ldots$ & 0.02 \\
\hline NGC7078 & 7.101 & 0.030 & 6.692 & 0.044 & 6.439 & 0.044 & 6.288 & 0.044 & 0.00 & 0.00 & 0.00 & 0.00 \\
\hline NGC7089 & 7.297 & 0.030 & 6.836 & 0.044 & 6.567 & 0.044 & 6.384 & 0.044 & 0.00 & 0.01 & 0.00 & 0.00 \\
\hline NGC7099 & 8.306 & 0.030 & $\ldots$ & $\ldots$ & $\ldots$ & $\ldots$ & 7.501 & 0.044 & 0.00 & $\ldots$ & $\ldots$ & 0.00 \\
\hline Pal12 & 12.489 & 0.030 & 11.826 & 0.043 & 11.482 & 0.044 & 11.345 & 0.045 & 1.38 & 1.24 & 1.15 & 1.06 \\
\hline Pal13 & 15.938 & 0.040 & 15.690 & 0.048 & 15.244 & 0.051 & 15.843 & 0.112 & 0.00 & 0.00 & 0.00 & 0.00 \\
\hline NGC7492 & 12.197 & 0.038 & 11.824 & 0.045 & 11.570 & 0.044 & 11.560 & 0.046 & 0.00 & 0.00 & 0.00 & 0.00 \\
\hline
\end{tabular}

